\title{
The EFT and FCNC interpretations in the processes with top quarks at CMS
}

\section{K. Skovpen (On behalf of the CMS Collaboration)*}

Vrije Universiteit Brussel, IIHE, Pleinlaan 2, 1050 Brussels, Belgium

E-mail: kirill.skovpen@cern.ch

In absence of any distinct evidence of new physics phenomena at the LHC, an increasing number of experimental studies aim at probing anomalous effects with an effective field theory (EFT) that represents a comprehensive approach for interpretation of various experimental results. The processes with the production of top quarks are sensitive to several classes of EFT operators including the flavour-changing neutral currents (FCNC). The summary of the latest CMS results based on the recent studies of the standard model processes and searches for new physics effects involving top quarks are presented.

European Physical Society Conference on High Energy Physics - EPS-HEP2019 -

10-17 July, 2019

Ghent, Belgium

* Speaker. 


\section{Introduction}

The standard model (SM) is incomplete. Nevertheless, we have not found any experimental evidence of new physics yet. It is possible that new particles that are considered in many beyond the SM (BSM) theories have masses that are above the energy reach at the Large Hadron Collider (LHC). The presence of new heavy particles can potentially induce various anomalous interactions at the electroweak and these effects can be studied at the LHC. Possible deviations from the SM predictions can be parametrized in a general way with an effective field theory (EFT) approach that uses an extended SM lagrangian built on dimension-six operators (SMEFT) [1, 2]. A full classification of the EFT operators relevant to the processes with top quarks is given in Ref. [3].

The so-called flavour-changing neutral currents (FCNC) are forbidden at tree level in the SM. These processes can only occur at higher orders and are highly suppressed due to the GlashowIliopoupos-Maiani (GIM) mechanism [4]. However, the FCNC transitions can be significantly enhanced by several orders of magnitude in various BSM scenarios [5]. The FCNC interactions with top quarks are particularly suppressed in the SM with the branching fractions of the top quark FCNC decays predicted at the level of $10^{-17}-10^{-12}$. In some of the best motivated BSM scenarios, such as the Two Higgs Doublet Model and the Minimal Supersymmetric Standard Model, these probabilities can be increased up to $10^{-10}-10^{-3}$. Such high rates can be probed in the ongoing experiments at the LHC, representing an excellent probe of BSM phenomena via a FCNC study.

\section{Analysis of dilepton events}

The processes with the production of a top quark in association with a W boson (tW) are associated with the final states containing two opposite-sign leptons. These final states also receive a significant contribution from the production of top quark pairs (tit). A simultaneous study of the tW and t⿱t processes in dilepton events was done at CMS [6] using $36 \mathrm{fb}^{-1}$ of $13 \mathrm{TeV}$ data [7]. This is the first study to directly constrain EFT in data using tW events. Both the tW and tit processes are sensitive to the chromomagnetic dipole moments $\left(\mathscr{O}_{\mathrm{tG}}\right)$. The tW production is also particularly sensitive to several EFT operators, such as the $\mathrm{Wtb}\left(\mathscr{O}_{\phi \mathrm{q}}^{(3)}\right.$ and $\left.\mathscr{O}_{\mathrm{tW}}\right)$ and the FCNC $\left(\mathscr{O}_{\mathrm{uG}}\right.$ and $\left.\mathscr{O}_{\mathrm{cG}}\right)$ couplings at the production. The triple gluon effective couplings $\left(\mathscr{O}_{\mathrm{G}}\right)$ contribute to the $\bar{t} \bar{t}$ production. For the effective couplings not involving FCNC interactions, any possible deviations from the SM predictions are dominated by the interference term between the SM and new physics diagrams, which is linear with respect to an EFT operator. For small values of the non-FCNC effective couplings the predicted distributions approach those of the SM processes, and therefore only the production rates of the $\mathrm{tW}$ and $\mathrm{t} \overline{\mathrm{t}}$ processes are used to derive the limits on the EFT Wilson coefficients. A multivariate analysis (MVA) approach is used to distinguish between the tW and $\bar{t}$ production processes, as well as to select FCNC events. One of the important systematic uncertainties in this analysis is associated with theoretical prediction of the studied processes. The measured EFT constraints on the Wilson coefficients are consistent with the SM predictions.

A study of spin correlations and differential cross sections of the t⿱t production is sensitive to the top quark chromomagnetic (CMDM, $\mathscr{O}_{\mathrm{tG}}$ ) and chromoelectric dipole moments (CEDM, $\mathscr{O}_{\mathrm{tG}}^{\mathrm{I}}$ ). The analysis of $36 \mathrm{fb}^{-1}$ of $13 \mathrm{TeV}$ CMS data focuses on the study of the properties of the spin density matrix as a function of the partonic initial state and production kinematics [8]. The density 


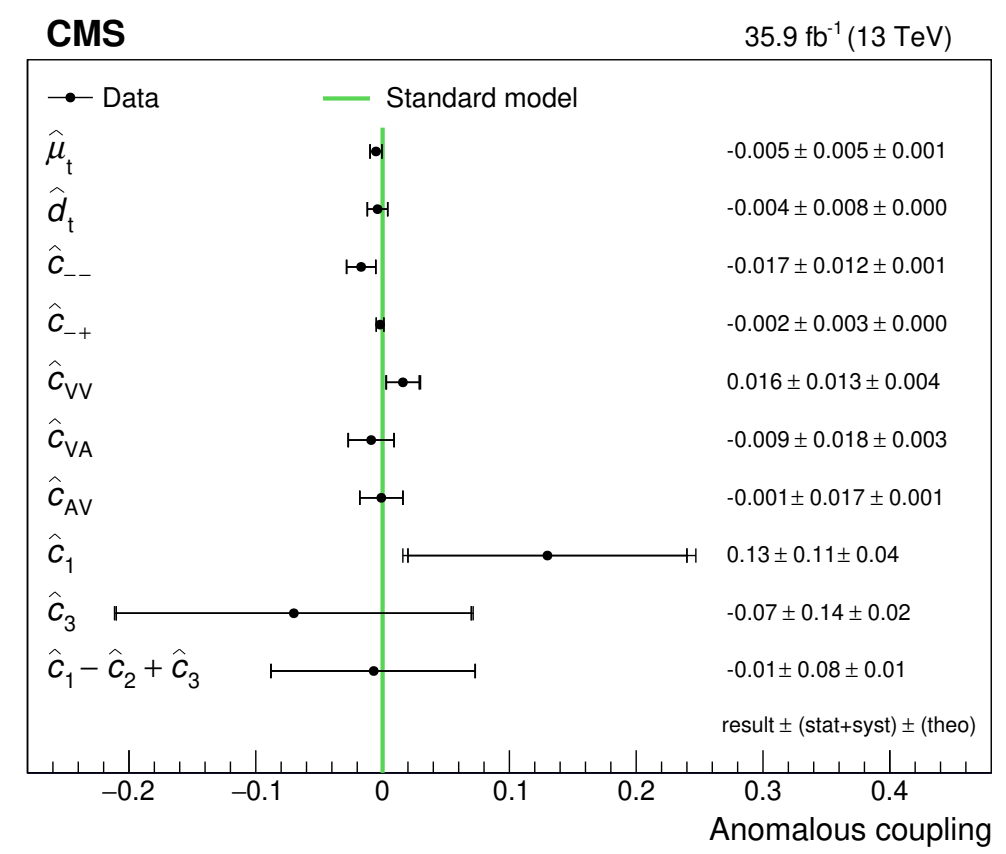

Figure 1: The measured constraints on the anomalous couplings of effective operators, including the chromomagnetic $\left(\hat{\mu}_{t}\right)$ and choromoelectric $\left(\hat{d}_{t}\right)$ dipole moments [8]. One non-vanishing Wilson coefficient is assumed at a time.

matrix is decomposed in a series of coefficients using the Pauli matrix basis to define experimental observables sensitive to the CMDM and CEDM. Due to the measurement of the full density matrix, the sensitivity to the CMDM and CEDM has been significantly enhanced with respect to the previously published direct constraints on these interactions. The modelling uncertainties associated with the simulation of the studied processes are among the dominant contributions to the total systematic uncertainty in this analysis. The resultant constraints on the studied anomalous effective couplings, including the four-quark and two-quark-gluon(s) operators, are presented in Fig. 1. The results also include two-dimensional limits on effective couplings.

\section{Search for the four top quark production}

The process with the production of four top quarks (ttttit) provides an important test of the QCD predictions and is also sensitive to the four-fermion EFT operators $\left(\mathscr{O}_{\mathrm{tt}}^{1}, \mathscr{O}_{\mathrm{QQ}}^{1}, \mathscr{O}_{\mathrm{Qt}}^{1}\right.$, and $\left.\mathscr{O}_{\mathrm{Qt}}^{8}\right)$. The study of this process uses $36 \mathrm{fb}^{-1}$ of $13 \mathrm{TeV}$ CMS data and is performed in a combination of several search channels: same-sign and opposite-sign dilepton, as well as single lepton and trilepton final states [9]. Potential new physics effects are probed in a restricted BSM scenario where it is assumed that new physics couples predominantly to the left-handed third generation quark doublet and the right-handed top quark singlet. The analysis uses the reconstruction of the hadronic top quark decays and defines several event categories based on the number of reconstructed jets. An MVA approach is used to suppress relevant background processes. The dominant uncertainties in the analysis are associated with the limited statistics in data, heavy-flavour jet identification, as well as jet reconstruction. The exclusion intervals on the EFT Wilson coefficients are extracted from the 
upper limit on the tttt production cross section. The resultant constraints are consistent with the SM predictions. The observed (expected) sensitivity to the tttt production was obtained at the level of 1.4 (1.1) standard deviations.

\section{Study of the associated production of top quarks with vector bosons}

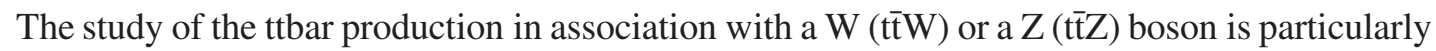
sensitive to the electroweak couplings of the top quark. These production processes also represent an important background in the study of the Higgs boson $(\mathrm{H})$ production in association with top

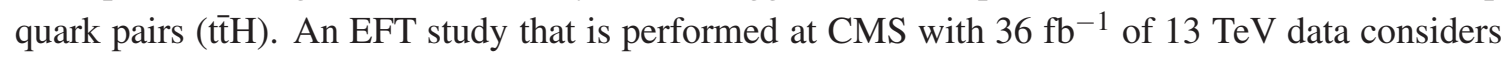
the $t \bar{t} \mathrm{~W}$ and $\mathrm{t} \bar{Z} Z$ production processes together with the contributions from the $\bar{t} \mathrm{t} H$ production [10]. From a large number of EFT operators potentially sensitive to the studied processes, only those are kept that are exclusively associated with an enhanced sensitivity to the $\bar{t} \mathrm{t} W$ and $\bar{t} \bar{Z} Z$ productions. Eight EFT operators were selected that are associated with the top quark electroweak couplings, as well as triple gluon field and the Higgs boson couplings. The analysis is done in the samesign dilepton, trilepton and four-lepton final states. Events are classified based on the number of additional reconstructed jets. The limits on the anomalous effective couplings are extracted from a combined likelihood fit over the defined event categories. The resultant constraints are in agreement with the SM predictions. The main systematic uncertainties are associated with the integrated luminosity, lepton identification, trigger selection efficiencies, and non-prompt lepton backgrounds.

The measurement of the differential tt̄ $Z$ production cross section is done at CMS using $78 \mathrm{fb}^{-1}$ of $13 \mathrm{TeV}$ data [11]. The analysis considers final states with three and four leptons. The EFT operators relevant to this study are the electroweak dipole moments $\left(\mathscr{O}_{\mathrm{tZ}}, \mathscr{O}_{\mathrm{tZ}}^{[\mathrm{I}]}\right)$ and anomalous neutral-current interactions $\left(\mathscr{O}_{\phi \mathrm{t}}, \mathscr{O}_{\phi \mathrm{Q}}^{-}\right)$. The analysis strategy and the dominant sources of systematic uncertainties are similar to Ref. [10]. The study uses a reweighting approach that is applied to the generator-level information to predict BSM effects at next-to-leading order with a proper treatment of systematic correlations. The statistical uncertainty is comparable to the total systematic uncertainty in this measurement. In addition to the EFT study, this analysis includes constraints on the vector and axial-vector current couplings, as well as the electroweak dipole moments. The final results are presented as one- and two-dimensional constraints on the effective couplings. A comparison of the resultant one-dimensional constraints on the Wilson coefficients to various direct and indirect studies is shown in Fig. 2.

\section{Search for FCNC with top quarks}

The FCNC processes with top quarks are studied in various search channels at CMS. The summary of the latest results is presented in Fig. 3.

A search for the top quark FCNC interactions with the Higgs boson is done for the case of the $\mathrm{H} \rightarrow \mathrm{b} \overline{\mathrm{b}}$ decays using $36 \mathrm{fb}^{-1}$ of $13 \mathrm{TeV}$ data [13]. This study considers top FCNC interactions in events with the production of top quark pairs, as well as in the associated production of a top quark and a Higgs boson. The latter is only sensitive to the top FCNC interaction with an up quark that is enhanced due to the proton parton distribution function. The analysis introduces several event 


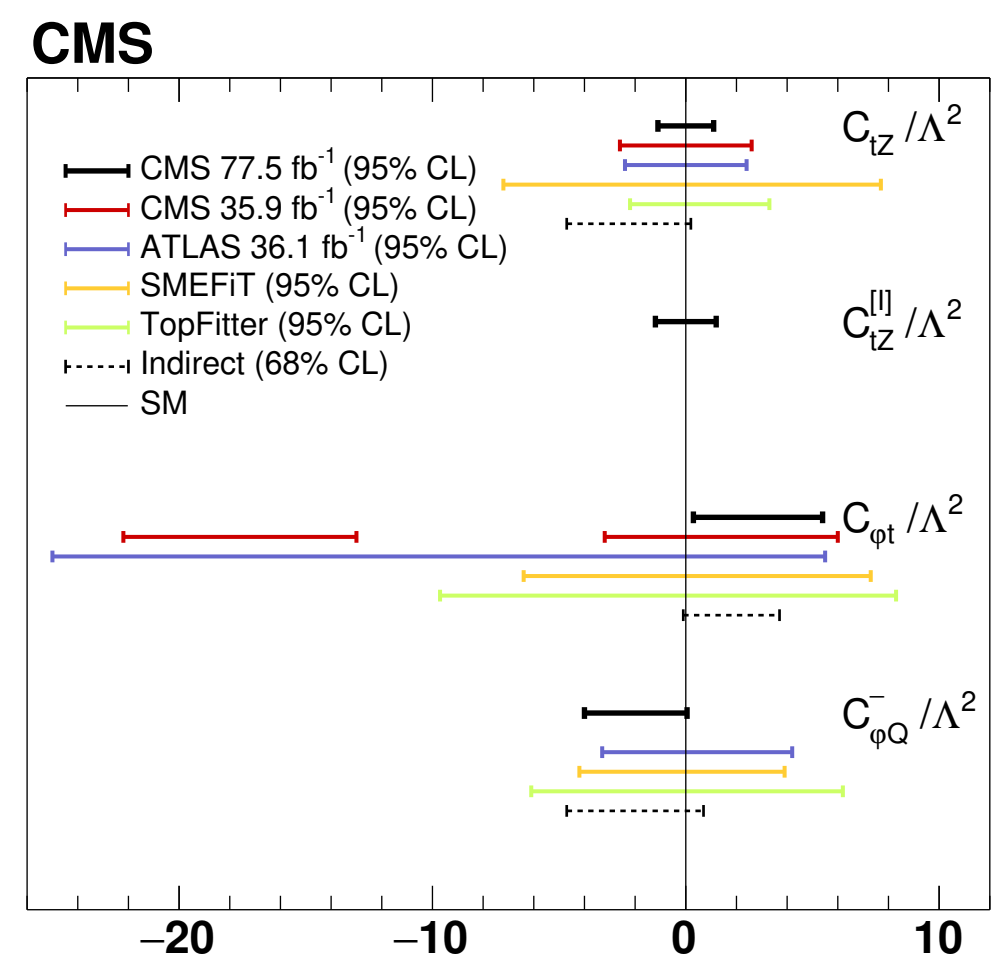

Figure 2: The observed constraints on the Wilson coefficients extracted from the differential cross section measurement of the tt̃ $Z$ process [11]. The results are compared to various previous experimental constraints and results of combined fits relevant to these operators.

categories defined by the number of reconstructed jets originating from an hadronization process of $b$ quarks. The main systematic uncertainty in this analysis is associated with the identification of heavy flavour jets. The extraction of final constraints on the top FCNC couplings is performed with a likelihood fit over the defined event categories. The observed (expected) contraints on the $\mathrm{t} \rightarrow \mathrm{Hu}$ and $\mathrm{t} \rightarrow \mathrm{Hc}$ branching fractions are $0.47 \%(0.34 \%)$ and $0.47 \%(0.44 \%)$, respectively.

A search for the top FCNC interactions with a $\mathrm{Z}$ boson is done in the final states with three leptons and is based on the analysis of $36 \mathrm{fb}^{-1}$ of data collected at $13 \mathrm{TeV}$ [14]. The FCNC effects are studied in the top quark decays and in the process of a top quark associated production with a $\mathrm{Z}$ boson. In the study of each of the production channels a signal kinematic region is defined using an MVA approach. The main systematic uncertainties are associated with non-prompt leptons and normalization of the background processes with the production of multiple $\mathrm{W}$ and $\mathrm{Z}$ bosons, as well as the $\bar{t} \mathrm{Z}$ production process. The FCNC constraints are extracted from a combined likelihood fit based on the distributions of the MVA discriminants obtained in the signal regions. The observed (expected) limits on the $\mathrm{t} \rightarrow \mathrm{Zu}$ and $\mathrm{t} \rightarrow \mathrm{Zc}$ branching fractions are $0.024 \%(0.015 \%)$ and $0.045 \%$ $(0.037 \%)$, respectively.

\section{Future prospects and summary}

Future studies of the EFT anomalous couplings and FCNC interactions in the top quark sector 


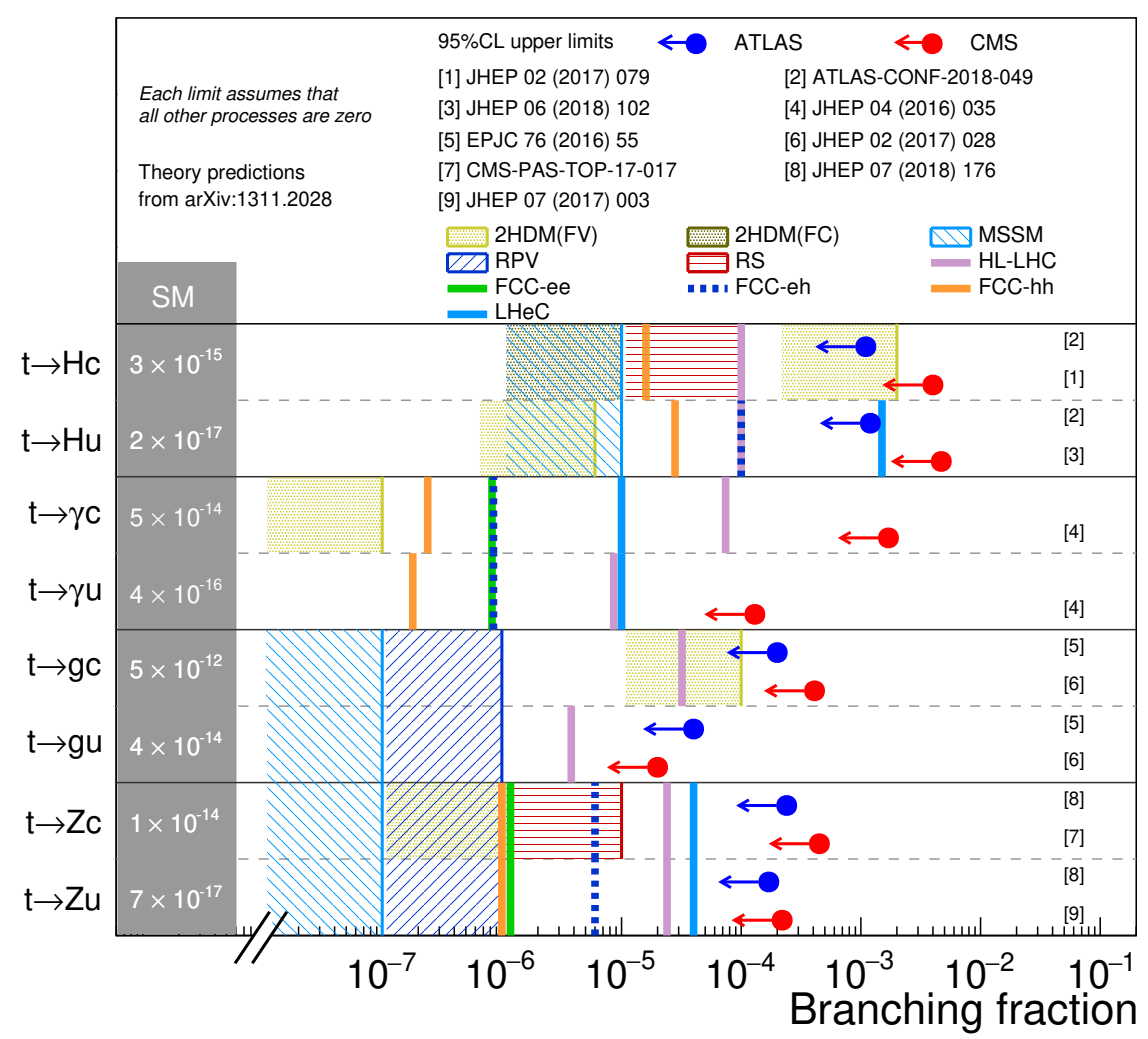

Figure 3: The present results and future projections for the limits on the top quark FCNC decay branching fractions [12]. The experimental constraints are compared to various BSM predictions [5].

at the High Luminosity LHC (HL-LHC) are associated with an important gain in the sensitivity

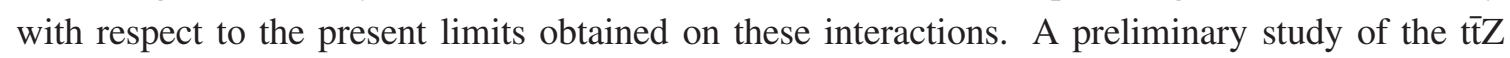
process at the HL-LHC resulted in the EFT constraints that are better by a factor of two than the current best limits obtained at the LHC $[15,16]$. The projected two-dimensional limits on the EFT Wilson coefficients are shown in Fig. 4. A similar level of improvement in constraining EFT operators is also anticipated in the analysis of the tttt production [17]. The HL-LHC data set will allow to further constrain the anomalous top FCNC decays. The projected sensitivities are expected to surpass by almost one order of magnitude the current best limits [12]. The future projections for the EFT studies of the FCNC processes with top quarks show an improvement of a factor of two in the final constraints on the two-fermion operators [18]

Over the past few years there has been an increasing number of experimental analyses in the top quark sector using an EFT approach to probe new physics effects. These studies provide an important set of constraints that can be easily reinterpreted for a given BSM model. A lot of progress was done with the full classification of the relevant EFT operators for the processes involving top quarks, providing an important guidance for various ongoing experimental searches at the LHC. The future studies at the HL-LHC are expected to significantly improve the current best limits on the EFT and FCNC couplings. 

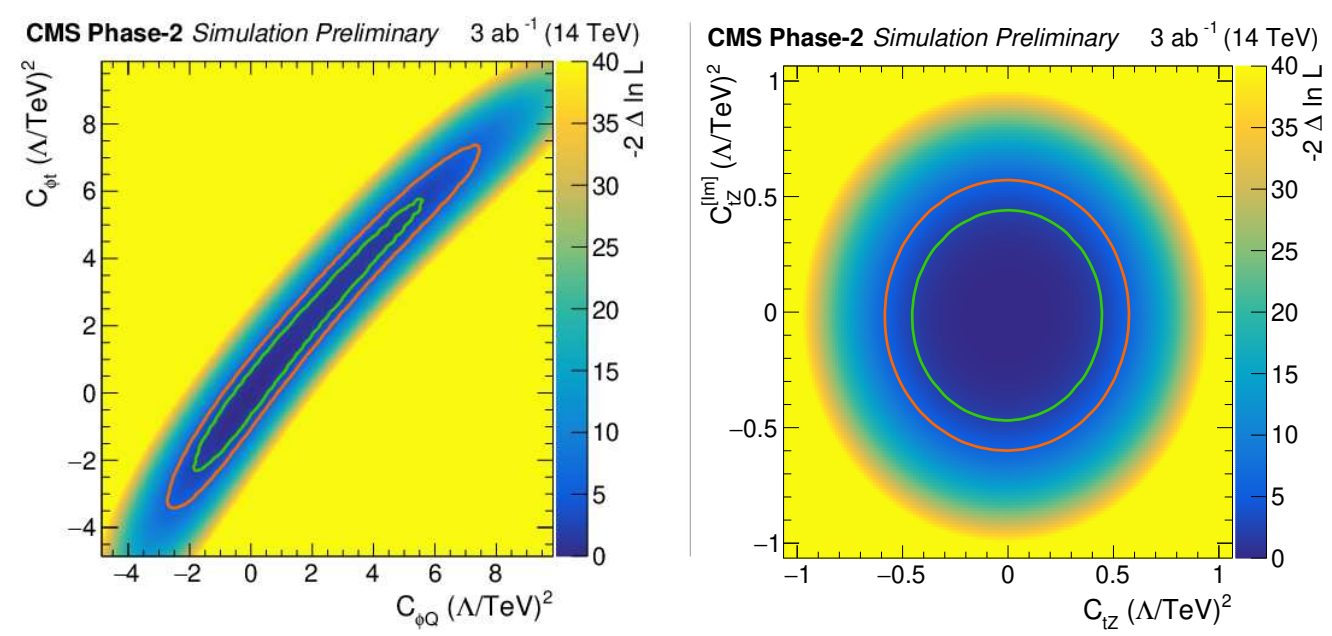

Figure 4: A negative likelihood as a function of the Wilson coefficients: $\mathscr{O}_{\phi \mathrm{Q}}^{-} / \mathscr{O}_{\phi \mathrm{t}}($ left $)$ and $\mathscr{O}_{\mathrm{tZ}} / \mathscr{O}_{\mathrm{tZ}} \mathrm{[ \textrm {I } ]}$ (right) [16]. The $68 \%$ and $95 \%$ confidence level contours are shown in green and red colours, respectively.

\section{References}

[1] W. Buchmuller and D. Wyler, Nucl. Phys. B 268 (1986) 621.

[2] B. Grzadkowski, M. Iskrzynski, M. Misiak et al., JHEP 10 (2010) 085.

[3] J. A. Aguilar-Saavedra et al., arXiv:1802.07237.

[4] S. L. Glashow, J. Iliopoulos, and L. Maiani, Phys. Rev. D 2 (1970) 1285.

[5] J. A. Aguilar-Saavedra, Acta Phys. Polon. B 35 (2004) 2695.

[6] CMS Collaboration, JINST 3 (2008) S08004.

[7] CMS Collaboration, arXiv:1903.11144 (Submitted to EPJC).

[8] CMS Collaboration, arXiv:1907.03729 (Submitted to Phys. Rev. D).

[9] CMS Collaboration, arXiv:1906.02805 (Submitted to JHEP).

[10] CMS Collaboration, JHEP 08 (2018) 011.

[11] CMS Collaboration, arXiv:1907.11270 (Submitted to JHEP).

[12] A. Abada et al., Eur. Phys. J. C 79 (2019) 474.

[13] CMS Collaboration, JHEP 06 (2018) 102.

[14] CMS Collaboration, CMS PAS TOP-17-017, http://cds.cern.ch/record/2292045.

[15] P. Azzi et al., arXiv:1902.04070.

[16] CMS Collaboration, CMS PAS FTR-18-036, http://cds.cern.ch/record/2652018.

[17] CMS Collaboration, CMS PAS FTR-18-031, http://cds.cern.ch/record/2650211.

[18] A. Cerri et al., arXiv:1812.07638. 